\title{
Minéralogie de la fraction argileuse des sols brunifiés de Kahankro et Anikro (Toumodi) dans le Centre Sud de la Côte d'Ivoire
}

\author{
K. E. YOBOUE*, A. J. BONGOUA-DEVISME, K. P. KOUADIO et A. YAO-KOUAME \\ UFR STRM, Département des Sciences du Sol, Université Félix Houphouët Boigny d'Abidjan Cocody, \\ Côte d'Ivoire. 22 BP. 582 Abidjan 22, Côte d'Ivoire. \\ *Auteur correspondant, E-mail : yobkemil@yahoo.fr
}

\section{RESUME}

Dans la perspective d'élucider les occurrences minéralogiques des sols brunifiés de Kahankro et de Anikro (Toumodi), dans la région centre de la Côte d'Ivoire, nous nous sommes intéressés à la fraction argileuse des sols. Cette étude, dans sa phase technique, a consisté à extraire la fraction argileuse des échantillons de sol prélevés sur le terrain en s'appuyant sur la méthode de Hotzapfel. Ces échantillons, de même que ceux du sol total, ont été ensuite soumis à une analyse aux rayons X sur lame orientée séchée à l'air puis, à des analyses thermodifférentielles et thermopondérales à une température allant jusqu'à $1200{ }^{\circ} \mathrm{C}$. Les résultats obtenus au bout de cette étude indiquent que les sols brunifiés de Kahankro et de Anikro contiennent différents types de minéraux existant en des proportions aussi variées. Il s'agit des oxydes (quartz, hématite, ilménite et boéhmite) avec une proportion généralement supérieure à 39\%, des minéraux primaires (feldspaths, olivine, dolomite et calcite) ayant une proportion supérieure à $30 \%$ et des minéraux argileux (kaolinite, illite et chlorite) qui existent en une proportion généralement inférieure à $25 \%$. Le phénomène d'argilation qui prévaut dans ces sols est la monosiallitisation, vu la prédominance de minéraux argileux de type 1/1 (kaolinite) sur les autres minéraux argileux. A cette monosiallitisation s'adjoint une bisiallitisation gouvernée par la richesse des sols en minéraux primaires. Ces sols sont aussi affectés par un phénomène de ferrallitisation et les paragenèses minérales de type kaolinite-hématite permettent de les définir comme étant caractéristiques d'une couverture d'altération mixte.

() 2014 International Formulae Group. All rights reserved.

Mots clés : Occurrences minéralogiques, phénomène d'argilation, ferrallitisation.

\section{INTRODUCTION}

Divers travaux réalisés sur différents sols par des auteurs tels que Doumbia et al. (2002) et Veyret et Vigneau (2002) ont montré que la qualité et l'abondance des différents éléments du sol dépendent pour une part importante de la nature chimique des minéraux constitutifs de la roche. $\mathrm{La}$ détermination de l'état actuel de l'évolution géochimique de la fraction minérale du sol permet de comprendre la genèse, l'utilisation et la classification des sols selon Pédro (2004). S'appuyant sur ces thèses, les travaux de Yao-Kouamé (2008) et de Yoboué et al. (2010), effectués sur les sols brunifiés de Toumodi, ont permis de mettre en évidence certaines évolutions pédogéochimiques depuis les roches jusqu'aux sols de la zone de Kahankro et de Anikro. En effet, les analyses chimiques effectuées sur le sol total et sur la 
fraction argileuse du sol ont mis en exergue la dynamique des éléments chimiques de la roche au sol et à la fraction argileuse. La détermination de la minéralogie de la fraction sableuse des sols, les analyses en éléments totaux de fer, aluminium, silicium, manganèse, magnésium, calcium, sodium, et potassium, ainsi que le calcul de rapports caractéristiques $\mathrm{SiO}_{2} / \mathrm{Al}_{2} \mathrm{O}_{3}$, laissent supposer l'existence d'une minéralogie très diversifiée et aussi très variée dans les sols sans toutefois donner plus de précisions sur la nature de ces minéraux. Cette suspicion pose la question de l'identité minéralogique ou des occurrences minéralogiques des sols brunifiés formés sur matériaux volcano-sédimentaires dans la zone subtropicale. Dans l'intention d'apporter une plus grande clarté à la minéralogie des sols brunifiés de Toumodi, nous avons axé cette étude sur la détermination des minéraux présents dans le sol total et dans la fraction argileuse du sol. Il s'agira dans ce cas, d'extraire la fraction argileuse du sol total en vue d'une analyse ultérieure à l'aide de méthodes appropriées pour une détermination minéralogique.

\section{MATERIEL ET METHODES}

L'étude minéralogique de la fraction argileuse du sol a d'abord commencé sur le terrain par une ségrégation de l'échantillonnage au niveau des profils de sol aux $20 \mathrm{~cm}$ selon les niveaux topographiques de sommet, de mi-versant et de bas de versant.

La méthode d'extraction de la fraction argileuse que nous avons adoptée se fonde d'une part, sur la méthode de séparation des argiles par la granulométrie utilisée dans les laboratoires de l'ORSTOM en 1981, et d'autre part sur les méthodes d'étude des argiles selon Holtzapffel (1985). Elle a donc consisté en la destruction de la matière organique, la dispersion ou défloculation par ajout de dispersant, la sédimentation, le prélèvement de surnageant, la décarbonatation (à $\mathrm{HCl} \mathrm{N} \mathrm{/}$ 5) et la centrifugation. La fraction argileuse obtenue a été soumise à une analyse aux rayons $\mathrm{X}$, échantillon séché à l'air et, à une analyse thermique.

Aux rayons $\mathrm{X}$, le diffractomètre utilisé est de type Philips PW opérant sous une tension de $35 \mathrm{kV}$ et à une intensité de $16 \mathrm{~mA}$. La radiation utilisée est la raie $\mathrm{K} \alpha$ de Cobalt (Co) avec un filtre de cuivre selon Jackson (1969). Les échantillons destinés à l'étude de rayons $\mathrm{X}$ ont été initialement passés au tamis à l'aide d'un crible de $0,125 \mathrm{~mm}$ de maille, puis montés sur une lame de verre et couverts d'une fine couche de vaseline. La vitesse de diffraction utilisée est de $2 \%$ min de 2 à 60 degrés. Après diffraction, on a obtenu un spectre d'énergie du faisceau diffracté en fonction de l'angle de diffraction $2 \theta$. Dans ce spectre, on a pu convertir les valeurs angulaires en espace basal d (en angströms) en appliquant la Loi de Bragg et en utilisant la longueur d'onde $\mathrm{K} \alpha$ de l'anode (Co) utilisée pour produire le rayonnement $\mathrm{X}$ incident selon Moore et Reynolds (1997). Les spectres de diffraction ou diffractogrammes obtenus pour les différents échantillons ont été interprétés via un logiciel de traitement. Pour l'identification des raies caractéristiques des différents minéraux, nous nous sommes appuyés sur les travaux effectués par certains auteurs comme Feder (2001), Alvarez-Puebla et al. (2004), Drits et al. (2004 et 2007), Mc Carty et al. (2008), Lanson et al. (2009), Hubert et al. (2009).

Les analyses thermiques ATD et ATG ont été effectuées grâce à un appareil ATD-ATG couplé de type SETARAM-TG 96. La vitesse de chauffage a été fixée à 5 ${ }^{\circ} \mathrm{C} / \mathrm{min}$; pour un intervalle de mesure compris entre 25 et $1400{ }^{\circ} \mathrm{C}$ et l'interprétation des résultats s'est appuyée sur les travaux de certains auteurs comme Viswabaskara et al. (2003), Ayari et al. (2004), et Kadiebu (2005). Ces méthodes, associées aux rayons $\mathrm{X}$, ont permis une identification rapide et fiable des phases minérales contenues dans les sols brunifiés de Kahankro et de Anikro. 


\section{RESULTATS \\ Minéraux argileux}

Les minéraux argileux identifiés sont essentiellement la kaolinite, l'illite et la chlorite.

- kaolinite : elle est caractérisée par les raies à 7,14 ̊́; 3,57 ̊́; 2,38 Á et 2,34 ̊̊ (Figure 1). Cette kaolinite est en des proportions très variables dans ces sols, comme en témoignent les amplitudes des pics observés sur les difractogrammes (de faibles amplitudes ou de très fortes amplitudes).

- illite : elle se caractérise par les raies à 10,32 Á; 10,22 Á; 10,12 Á ; 10,02 Á; 9,98 Á ;

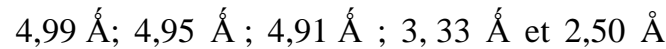
dont quelques unes sont présentées à la Figure 1. Les pics de l'illite sont parfois larges et /ou asymétriques. Il s'agit donc d'une illite mal cristallisée provenant de la décomposition d'un mica dont les feuillets s'ouvrent pour libérer dans le milieu pédologique du potassium $\left(\mathrm{K}^{+}\right)$.

- chlorite : elle est rencontrée seulement dans le sol de bas de versant à caractère vertique de Kahankro. Elle est identifiée par les raies à $14,63 \AA$ et celles à 7,12 Á (Figure 2). Le pic à $14,63 \AA$ Á est large et traduit de ce fait la mauvaise cristallinité de ce minéral ou qu'il existe en des formes variées (ferreuse ou alumineuse).

\section{Oxydes et autres minéraux oxydes}

- Quartz: le quartz est caractérisé par

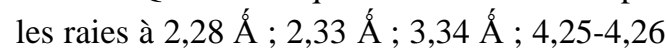
A. Ces pics sont très allongés, proportionnellement à la forte teneur de ce minéral dans le sol (Figures 1 et 2);

- Hématite : elle est caractérisée par les raies à 2,68-2,70 Á et 2,21 $\AA$; 2,17-2,18 Á. Mais la

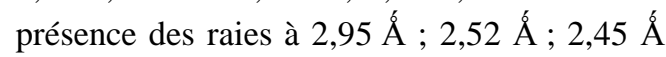
de la maghémite et des raies à 2,17-2,18 $\AA$; 2,24 ̊́; 4,16 ̊́ et 4,95-4,96 ̊̊; 4,98Á de la goethite permet de dire que l'hématite dans ces sols est souvent mal cristallisée, et existe sous forme d'oxyde hydraté de fer (Figures 1 et 2) ;

- Ilménite : l'ilménite se caractérise par les

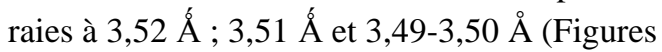
1 et 2$)$;

- Boéhmite : elle est caractérisée par les raies

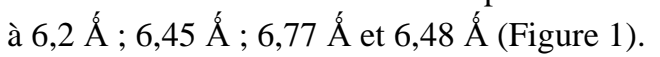

\section{Autres minéraux}

- Feldspath: au niveau des feldspaths, nous distinguons les raies caractéristiques des

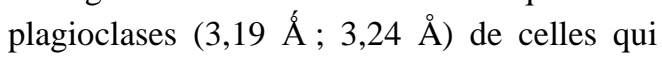
caractérisent les feldspaths potassiques

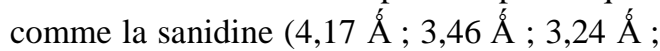

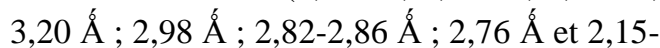
$2,16 \AA$ ) dont quelques unes sont présentées à la Figure 1. Toutes ces raies sont caractérisées par des pics d'amplitudes variables traduisant des quantités aussi variables de ces minéraux;

- Olivine : l'olivine se caractérise par diverses raies correspondant à diverses valeurs de $2 \theta$. Il

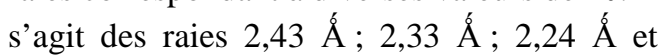
2,52 Á (Figure 2);

- Dolomite : elle est caractérisée par la raie 2,89-2,90 Á (Figure 1);

- Calcite : elle est déterminée par les raies de 2,11 Á à 2,13 $\AA$ et de 3,01 Á. On distingue aussi des raies à 2,28 $\AA$ qui sont à la fois caractéristiques du quartz (Figure 1).

Dans certains cas, les raies sont caractéristiques de plusieurs minéraux. Il s'agit par exemple de la raie 4,45 $\AA$ caractérisant la kaolinite, l'illite et même la vermiculite, de la raie $2,56 \AA$ de la kaolinite et de l'hématite, de la raie $2,28 \AA$ de la calcite et du quartz. Seules des études plus approfondies telles que le traitement à l'alcool et le chauffage pourront permettre de faire des séparations franches.

\section{Composition minéralogique globale des échantillons de sol}

L'analyse de diffraction de rayons $\mathrm{X}$ a été réalisée sur 12 échantillons de sol et 12 échantillons de fraction argileuse extraite du sol. Ces échantillons ont été soumis à l'interprétation minéralogique à un niveau 
semi quantitatif. Le modèle de rayons $\mathrm{X}$ obtenu à partir des scans de diffraction des échantillons orientés a été utilisé pour l'identification des minéraux argileux et des autres minéraux (oxydes et minéraux primaires) suivant les critères établis par Whittig et Jackson (1955), Brown (1961) et par Black et al. (1965). Les proportions des différents minéraux mis en évidence sont consignées dans le Tableau 1.

Concernant la présence des trois minéraux argileux (kaolinite, illite, et chlorite) dans les échantillons de sol, les résultats montrent qu'elle est dominée par la kaolinite (même si elle existe parfois en trace), dont les teneurs varient du modéré (de 15-25\%) au moyen (25-40\%). L'Illite est détectée dans presque tous les échantillons, elle existe en traces ou en faibles quantités (15-25\%). La chlorite est en traces $(<5 \%)$ et n'existe seulement dans le sol de bas de versant, à caractère vertique, de Kahankro.

Pour ce qui est des oxydes et des minéraux primaires, ils sont dominés respectivement par le quartz et les feldspaths. Ces minéraux sont détectés dans des quantités variables. Les teneurs en quartz varient des faibles quantités (5\%) aux quantités dominantes (> 40\%), tandis que les teneurs en feldspath varient des traces à des quantités modérées (15-25\%). Ensuite, viennent la dolomite et l'olivine, qui sont également détectés en traces ou en petites quantités dans tous les échantillons. Les quantités d'ilménite et de boéhmite varient des traces aux modérées, tandis que la calcite, l'hématite, et la hornblende sont en traces.

Mais la quantification des teneurs en ces différents groupes minéralogiques montre que les sols brunifiés de Kahankro et de Anikro sont plus riches en minéraux primaires et en oxydes qu'en minéraux argileux, quelle que soit la position topographique, si on s'en tient aux proportions de ces différents groupes minéralogiques :
- teneurs en oxydes > 39\% ;

- teneurs en minéraux primaires $>30 \%$;

- teneurs en minéraux argileux $<25 \%$.

\section{Analyses thermiques : ATD et ATG}

Pour appuyer les résultats obtenus aux $\mathrm{RX}$, nous avons effectué en complément, des analyses thermiques sur la fraction argileuse du sol. Les températures de chauffe auxquelles les échantillons ont été soumis se sont limitées à $1200{ }^{\circ} \mathrm{C}$. Les données recueillies au cours de ce chauffage nous ont permis de tracer les courbes ATD-ATG illustrées par l'exemple de celles du profil de sol de sommet à Kahankro de la Figure 3. L'analyse de ces courbes fait ressortir les observations suivantes :

- les courbes d'ATD présentent des pics endothermiques et des pics exothermiques à diverses températures de chauffe de l'échantillon :

$\checkmark \quad$ un pic endothermique, de faible intensité, entre $200-300{ }^{\circ} \mathrm{C}$. Ce pic caractérise le départ d'eau d'hydratation de la goethite et, dans une moindre mesure, de minéraux comme l'illite, la chlorite ferrifère et des smectites suite à des impuretés ;

$\checkmark \quad$ un pic endothermique, de plus forte intensité, entre $400-550{ }^{\circ} \mathrm{C}$, qui est caractéristique de la déhydroxylation de la kaolinite, de l'illite, de la chlorite alumineuse et des smectites ;

$\checkmark \quad$ un pic exothermique à environ 900

${ }^{\circ} \mathrm{C}$, faisant suite à la transformation de la kaolinite en mullite $2 / 1$ et en spinelle qui sont des minéraux de recristallisation, c'està-dire de néogenèse.

- les courbes d'ATG présentent, quant à elles, consécutivement aux réactions thermiques, des pertes de poids d'environ 2 et 6 ou $8 \%$ respectivement entre $200-300{ }^{\circ} \mathrm{C}$ et entre $400-550^{\circ} \mathrm{C}$. Ceci est généralement observé dans tous les profils des deux localités. Ces pertes de poids sont caractéristiques de l'illite, de la vermiculite et des smectites 
K. E. YOBOUE et al. / Int. J. Biol. Chem. Sci. 8(3): 1269-1280, 2014

Tableau 1: Composition minéralogique des échantillons de sol total et de la fraction argileuse.

\begin{tabular}{|c|c|c|c|c|c|c|c|c|c|c|c|c|}
\hline \multirow{2}{*}{$\begin{array}{l}\text { Références } \\
\text { échantillons }\end{array}$} & \multicolumn{9}{|c|}{ Minéraux accessoires } & \multicolumn{3}{|c|}{ Minéraux argileux } \\
\hline & Dolomite & Olivine & Hématite & Boéhmite & Ilménite & Hornblende & Calcite & Quartz & Feldspaths & Chlorite & Illite & Kaolinite \\
\hline $\mathrm{A}_{\mathrm{fa}}(\mathrm{S})(40-60)$ & ++ & ++ & ++ & ++ & ++ & ++ & ++ & ++ & +++ & - & + & +++ \\
\hline $\mathrm{A}_{\mathrm{fa}}(\mathrm{S})(80-100)$ & ++ & ++ & + & + & ++ & + & + & +++++ & ++ & - & - & ++ \\
\hline $\mathrm{A}_{\mathrm{fa}}(\mathrm{V})(20-40)$ & + & + & - & ++ & ++ & - & - & ++++ & ++ & - & - & ++++ \\
\hline $\mathrm{A}_{\mathrm{fa}}(\mathrm{V})(60-80)$ & ++ & + & +++ & - & + & + & + & +++++ & ++ & - & ++ & +++ \\
\hline $\mathrm{A}_{\mathrm{fa}}(\mathrm{BV})(0-20)$ & ++ & - & + & ++ & ++ & - & - & + & +++ & - & - & ++++ \\
\hline $\mathrm{A}_{\mathrm{fa}}(\mathrm{BV})(20-40)$ & ++ & + & ++ & - & + & + & + & +++++ & +++ & - & - & ++ \\
\hline $\mathrm{K}_{\mathrm{fa}}(\mathrm{S})(60-80)$ & + & + & - & ++ & ++ & + & + & ++++ & +++ & - & - & +++ \\
\hline $\mathrm{K}_{\mathrm{fa}}(\mathrm{S})(100-120)$ & ++ & ++ & - & ++ & ++ & - & + & +++++ & +++ & - & - & ++ \\
\hline $\mathrm{K}_{\mathrm{fa}}(\mathrm{V})(60-80)$ & ++ & ++ & - & + & - & - & + & +++++ & +++ & - & ++ & ++ \\
\hline $\mathrm{K}_{\mathrm{fa}}(\mathrm{V})(100-120)$ & ++ & + & ++ & ++ & +++ & - & ++ & ++ & +++ & - & - & +++ \\
\hline $\mathrm{K}_{\mathrm{fa}}(\mathrm{BV})(20-40)$ & + & + & ++ & ++ & + & + & - & +++ & ++ & - & - & +++++ \\
\hline $\mathrm{K}_{\mathrm{fa}}(\mathrm{BV})(40-60)$ & + & + & ++ & ++ & ++ & ++ & + & ++++ & ++ & - & + & ++ \\
\hline $\mathrm{A}_{\mathrm{st}}(\mathrm{S})(40-60)$ & ++ & + & - & + & + & + & + & +++++ & + & - & ++ & + \\
\hline $\mathrm{A}_{\mathrm{st}}(\mathrm{S})(80-100)$ & ++ & + & - & + & + & + & + & +++++ & + & - & + & + \\
\hline $\mathrm{A}_{\mathrm{st}}(\mathrm{V})(20-40)$ & ++ & + & + & + & + & - & + & +++++ & ++ & - & - & ++++ \\
\hline $\mathrm{A}_{\mathrm{st}}(\mathrm{V})(60-80)$ & ++ & ++ & + & - & + & - & ++ & +++++ & +++ & - & + & + \\
\hline $\mathrm{A}_{\mathrm{st}}(\mathrm{BV})(0-20)$ & ++ & + & - & + & ++ & - & + & ++++ & ++ & - & + & ++++ \\
\hline $\mathrm{A}_{\mathrm{st}}(\mathrm{BV})(20-40)$ & ++ & ++ & - & - & + & + & + & +++++ & + & - & + & + \\
\hline $\mathrm{K}_{\mathrm{st}}(\mathrm{S})(60-80)$ & ++ & ++ & - & + & + & + & + & +++++ & + & - & - & + \\
\hline $\mathrm{K}_{\mathrm{st}}(\mathrm{S})(100-120)$ & ++ & + & - & - & + & ++ & + & +++++ & + & - & + & + \\
\hline $\mathrm{K}_{\mathrm{st}}(\mathrm{V})(60-80)$ & ++ & + & + & - & + & + & + & +++++ & + & - & - & + \\
\hline $\mathrm{K}_{\mathrm{st}}(\mathrm{V})(100-120)$ & ++ & ++ & + & + & + & + & + & +++++ & + & - & + & + \\
\hline $\mathrm{K}_{\mathrm{st}}(\mathrm{BV})(20-40)$ & ++ & ++ & + & + & + & + & - & +++++ & + & - & - & + \\
\hline $\mathrm{K}_{\mathrm{st}}(\mathrm{BV})(40-60)$ & ++ & ++ & + & + & + & + & - & +++++ & + & + & - & - \\
\hline
\end{tabular}

烈 total, $\mathrm{S}=$ Sommet, $\mathrm{V}=$ Mi-Versant, $\mathrm{BV}=$ Bas de Versant, Les nombres entre parenthèses sont en $\mathrm{cm}$; ce sont les profondeurs de prélèvement des échantillons. 


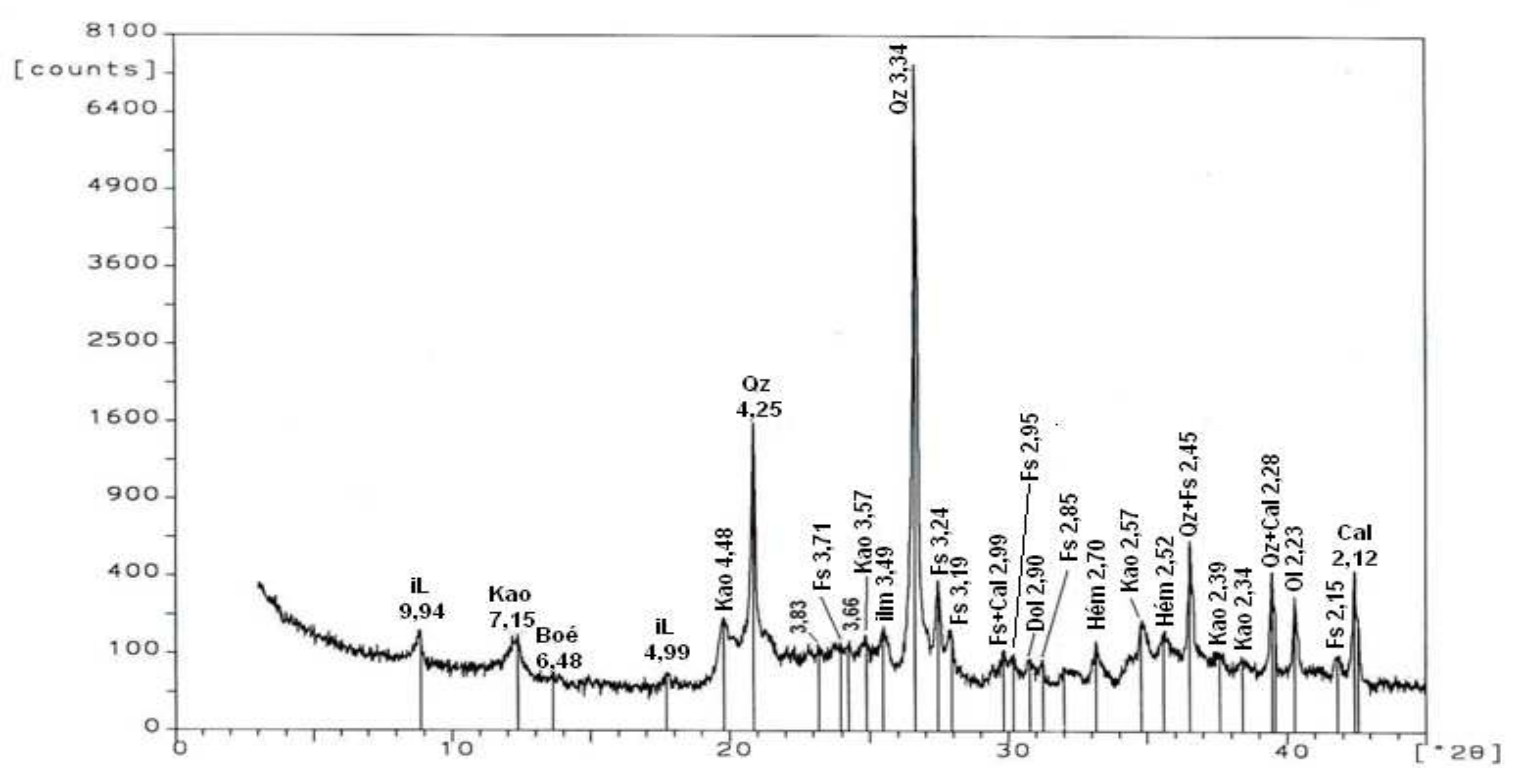

Figure 1: Difractogramme de rayons $X$ dans l'échantillon de sol total du profil de sol de sommet à Anikro dans la profondeur 40-60 cm. Kao = kaolinite; $\mathrm{Il}=$ illite ; Fs = Feldspath ; $\mathrm{Qz}=\mathrm{Quartz}$; Cal = Calcite ; $\mathrm{Hbl}=$ Hornblende $; \mathrm{Ilm}=$ Ilménite $;$ Boé = Boéhmite $; \mathrm{Hém}=$ Hématite $; \mathrm{Ol}=$ Olivine $; \mathrm{Dol}=$ Dolomite.

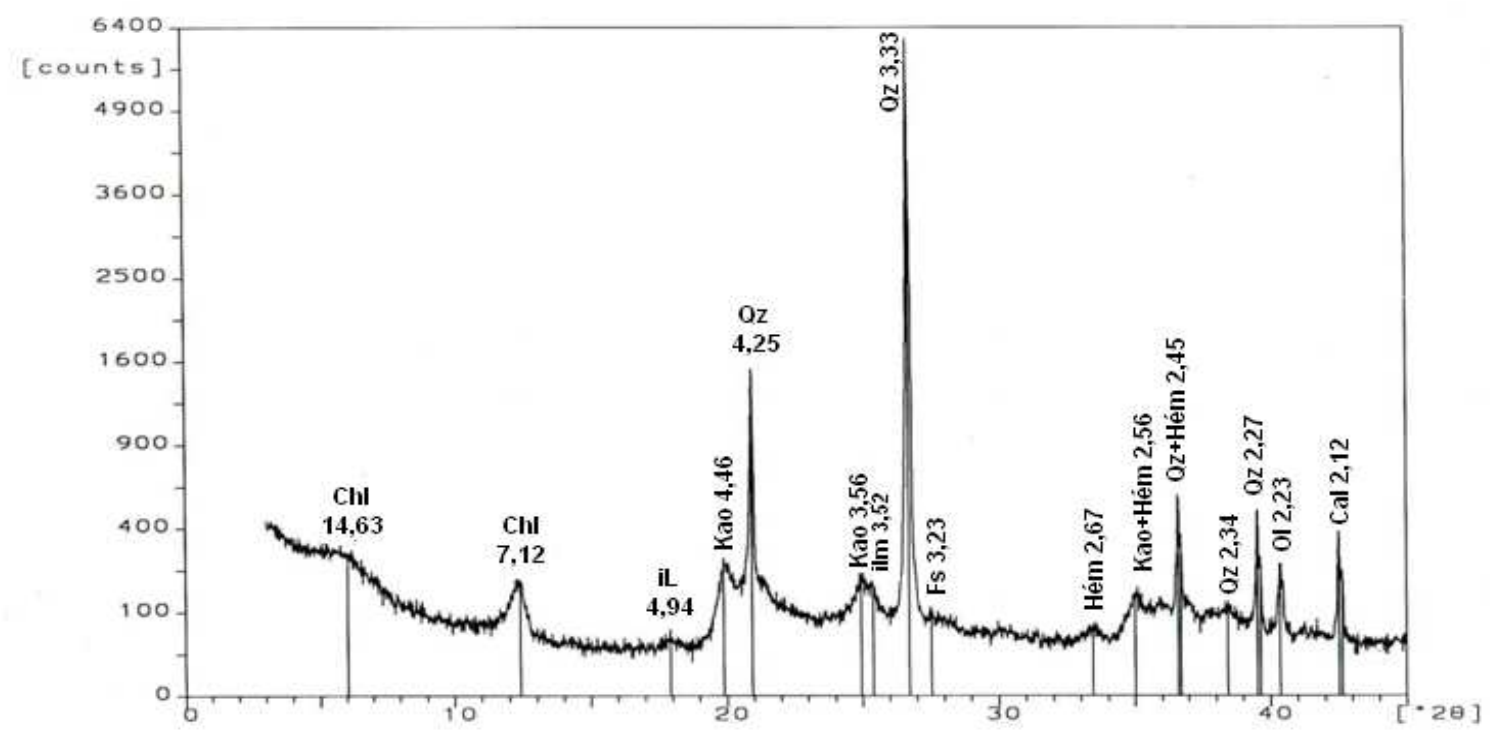

Figure 2: Difractogramme de rayons $X$ dans l'échantillon de sol total du profil de sol du bas de versant à Kahankro dans la profondeur 40-60 cm. Chl = Chlorite ; Kao = kaolinite ; $\mathrm{Il}=$ illite ; Fs = Feldspath ; Qz = Quartz; Cal = Calcite ; Hbl = Hornblende ; Ilm = Ilménite ; Boé $=$ Boéhmite ; Hém = Hématite ; Ol = Olivine ; Dol = Dolomite. 


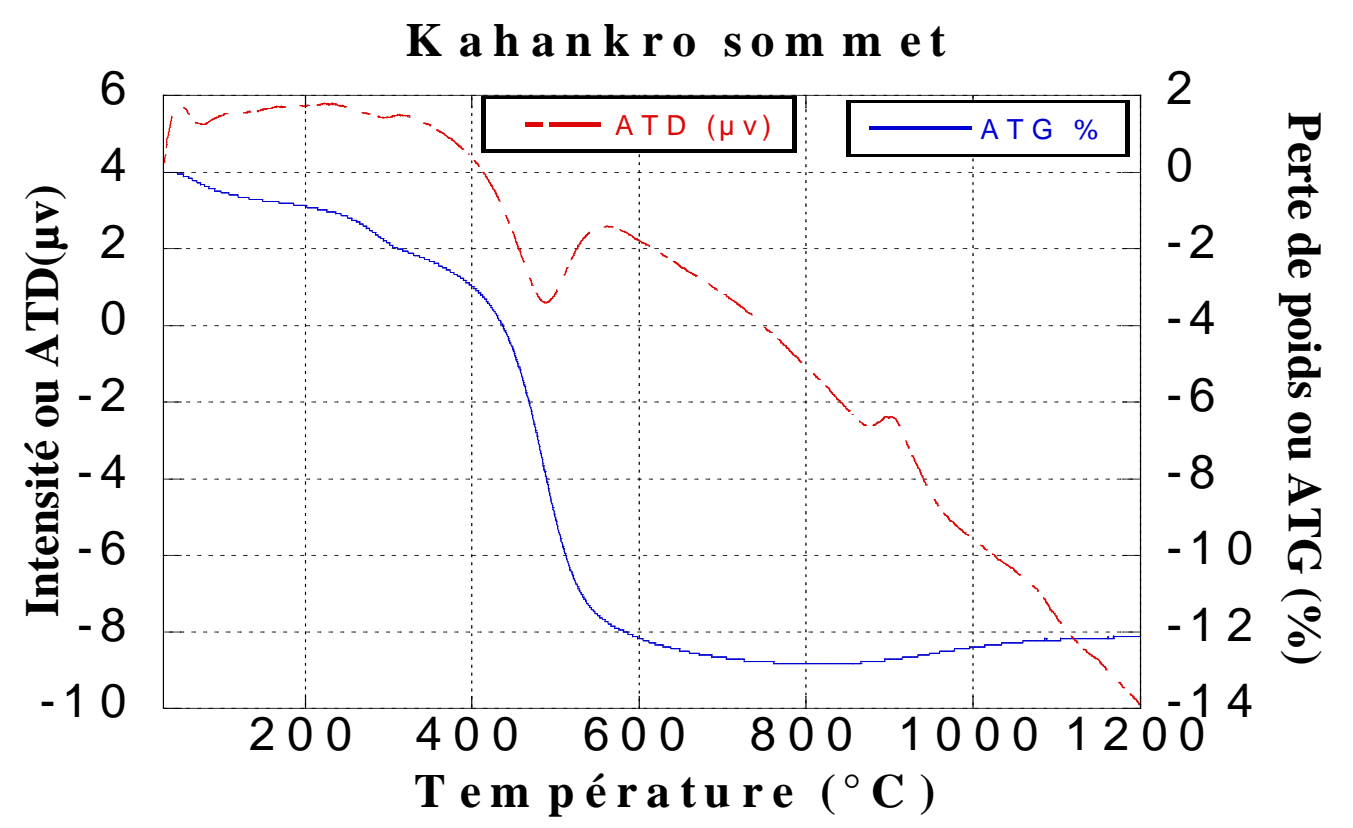

Figure 3: Courbes ATD-ATG dans le profil de sommet à Kahankro.

\section{DISCUSSION}

A partir des analyses aux rayons $\mathrm{X}$ (Figures 1 et 2 ) et des analyses thermiques (Figure 3), nous pouvons retenir que dans les cambisols de Toumodi, les minéraux argileux sont essentiellement de la kaolinite et de l'illite, auxquels s'ajouteraient des vermiculites, des chlorites et des minéraux du groupe des smectites. Cette prédominance de la kaolinite et l'absence quasi-totale de la gibbsite pourrait s'expliquer par une compensation plus ou moins importante de la perte de silice. En réalité, puisque l'altération se poursuit, il y a aussi desilification de la kaolinite qui devrait évoluer vers des gibbsites. Mais, dans le cas d'espèce, où il y a abondance de kaolinite sans gibbsite, nous pensons que cela aurait son origine dans la richesse des roches et des sols, en silice. En effet, les études effectuées dans la région par Yoboué et al. (2010), révèlent un développement impressionnant de silice dans des roches aussi basiques et, de plus, les travaux réalisés par Grimaldi (2004) montrent que lorsque le matériau est riche en silice, le renouvellement du stock de silice dissoute dans l'eau se fait toujours et l'on a des sols riches en kaolinite plutôt qu'en gibbsite. Mais, nous pensons aussi qu'en dehors de tout caractère siliceux des roches, cette prédominance de la kaolinite sur les minéraux argileux 2/1 serait aussi soutenue par le $\mathrm{pH}$ moyennement acide de ces sols tels que montré par Yoboué et al. (2010). Selon les études de Duchaufour (2001) et de Caner (2004), dans les horizons des sols tropicaux dont les pH se situent aux alentours de 5, et dont une partie des bases et de la silice a été évacuée par drainage profond, seules les argiles les plus pauvres en silice, de type kaolinite peuvent prendre naissance.

La présence des argiles 2/1 est favorisée par celle des minéraux primaires qui existent en fortes proportions dans ces sols. En effet, selon les études de Brown et al. (2003), la présence de minéraux primaires favorise la libération de cations bivalents $\mathrm{Ca}^{2+}$ et $\mathrm{Mg}^{2+}$, qui jouent un rôle important dans la 
formation d'argiles riches en silice. Cela a été confirmé par les travaux de Ben Hassine (2006) qui ont montré que la présence de telles argiles découle de l'altération des minéraux primaires hérités des roches. La présence de l'illite est donc favorisée par la forte composition du sol en minéraux primaires, car ces derniers, subissant toujours l'altération, libèrent dans le sol des ions métalliques basiques, dont la présence dans le sol est favorable à la synthèse de minéraux argileux de type mica, qui sont présents à titre transitoire. Cette composition minéralogique soutient encore l'idée d'un sol où prédomine un processus d'altération monosiallitique bien qu'existe encore un processus bisiallitique.

En fait, les minéraux des sols de Kahankro et de Anikro peuvent être regroupés d'une part en minéraux hérités (quartz, olivine, calcite, feldspath, hornblende) provenant des roches observées sur le terrain, et d'autre part en minéraux de néoformation qui proviennent du processus d'altération hydrolytique (argiles et oxy-hydroxydes de fer et d'aluminium). Tous ces minéraux observés dans le sol proviennent des minéraux ferromagnésiens, des minéraux calciques, des feldspaths et des oxydes opaques observés en lames minces dans les roches tel que montré par Yoboué et al. (2010). Dans les sols brunifiés de Kahankro et de Anikro, nous sommes bien en mesure de dire que l'hydrolyse est partielle par l'absence de la gibbsite, et que la désalcalinisation est quasiment totale, vu la prédominance de la kaolinite sur tous les autres minéraux argileux (illite, chlorite).

La kaolinite observée dans ces sols proviendrait de l'altération des plagioclases ou de feldspaths potassiques observés dans les roches sur lame mince. L'illite provient de la transformation des micas par perte progressive $\mathrm{du}$ potassium interfoliaire et pourrait de ce fait exister sous la forme d'interstratifié muscovite-illite. La formation des hydroxydes et oxydes de fer fait suite à l'altération de minéraux riches en fer comme l'olivine, les pyroxènes et la hornblende. En effet, en période humide, le fer provenant des minéraux ferromagnésiens est mis en mouvement dans le sol sous forme de fer ferreux $\left(\mathrm{Fe}^{2+}\right)$. En période sèche, l'absence d'eau entraîne l'existence du fer sous sa forme ferrique $\left(\mathrm{Fe}^{3+}\right)$, favorable à la formation des nodules et des concrétions comme montré par les travaux de Temgou et al. (2002), Van Ranst et De Coninck (2002) Oh et Richter (2005). Nous attribuons la présence de la silice au quartz issu de la microdivision de la roche, car, selon Ulery (2002) les pH acides (tels que ceux des sols étudiés) et le milieu intertropical sont favorables à une solubilisation plus poussée de la silice amorphe provenant de l'altération des silicates par rapport à celle du quartz.

Comme nous pouvons le constater, la constitution argileuse dans les cambisols de Toumodi est hétérogène. Elle est formée de trois types de minéraux argileux (kaoliniteillite-chlorite) auxquels s'ajoutent la vermiculite et les smectites révélés essentiellement par les analyses thermiques. Nous savons que la caractérisation des différentes couvertures pédologiques résulte essentiellement de l'étude de la fraction argileuse des sols ; mais en tout état de cause, elle ne repose que sur la nature des argiles néoformées. Ainsi, la prédominance de la kaolinite et la paragenèse kaolinite-illite sont caractéristiques d'une couverture d'altération siallitique mixte d'après les travaux de Pedro (2004) appuyés de ceux de Legros (2007). Selon ces auteurs, la présence des minéraux primaires, témoin d'un degré d'altération incomplet, milite en faveur d'une telle couverture d'altération. Par ailleurs, la présence des oxydes ferrifères (hématites), définissant la paragenèse kaolinite-hématite, permet de dire qu'il y a également dans ce sol un processus de ferrallitisation. Ce qui est tout à fait évident; puisque, en position 
topographique de sommet et de mi-versant, les sols bruns (cambisol) évoluent vers un sol ferrallitique (ferralsol) selon les travaux de Duchaufour (2001) et de Horbe et al. (2004). Dès lors, nous sommes bien en mesure de dire que le processus de ferrallitisation qui prévaut dans les sols environnants a dû affecter la genèse des cambisols de Toumodi.

La présence de minéraux primaires, signalée dans ces sols brunifiés, ne parait pas être un fait nouveau dans la composition minéralogique des sols bruns (cambisol). En effet, d'après les travaux de Duchaufour (2001), les sols bruns contiennent des minéraux altérables. Aussi, attribuons-nous la présence des minéraux primaires aux forts phénomènes de remaniement et de rajeunissement (Aleskeev et al., 2003), aboutissant parfois à l'amincissement des épaisseurs des sols observés sur le terrain, d'après les travaux de Yao-Kouamé (2008). Selon Darboux et Le Bissonnais (2007), ces modifications de la surface se font au fil des années. De plus, selon les études de Mallam et al. (2004), ces modifications de la surface affectent les phénomènes d'infiltration liées au développement de la croûte. Nous pensons que c'est bien pour cela que le rajeunissement s'accompagne d'un tachement des horizons du sol. Pour nous, la présence des minéraux primaires confirme encore que ces sols sont jeunes, donc peu évolués, suite aux caractères pétrographiques des roches observées sur le terrain et au climat tropical peu arrosé que celui des régions du Sud de la Côte d'Ivoire. Les roches ont parfois un caractère pyroclastique basique (amphibolites), avec des minéraux souvent visibles à l'œil nu, ou sont plus ou moins acides (dacites) comme montrés par Yoboué et al. (2010). Cela rejoint les travaux de Legros (2007) qui attribue la présence des minéraux primaires au fait que les sols soient jeunes, ou soumis à un climat moins agressif, ou encore au fait qu'ils sont formés sur matériaux pyroclastiques plus grossiers ou moins riches en verres. La prédominance des minéraux feldspathiques dans la fraction des minéraux primaires traduit le fait que ce minéral, tout comme le quartz, n'est pas facilement altérable (Campy et Macaire, 2003).

Cette forte présence des minéraux primaires dans les sols brunifiés de Toumodi constitue une source de fertilité absolue, car selon Quantin et al. (2001), les minéraux primaires contribuent à enrichir le sol en éléments nutritifs pour les plantes, notamment en bases, tout en favorisant la biodiversité microbienne du sol. Le comportement des cultures établis sur ces sols en témoigne éloquemment, vu que les essais de culture mis en place par Yao-Kouamé (2008) ont montré des plantes plus vigoureuses sur ces sols que sur les ferralsols situés dans le voisinage. Ce qui permet de vérifier que les caractéristiques minéralogiques de ces cambisols de Toumodi constituent un atout en termes de fertilité foncière.

\section{Conclusion}

La minéralogie de la fraction argileuse des sols brunifiés de Kahankro et de Anikro parait très riche en ce sens qu'on y observe à la fois des minéraux argileux, des oxydes et des minéraux primaires.

Le phénomène d'argilation qui accompagne l'altération des roches et des sols est essentiellement la monosiallitisation, avec formation préférentielle de kaolinite et d'illite. Cette paragenèse fait des sols brunifiés de Anikro et de Kahankro des couvertures d'altération siallitique mixtes qui sont aussi affectées par la ferrallitisation suite à la présence de l'hématite. Cette ferrallitisation est donc le fait de caractères minéralogiques et géochimiques de ces sols et soutien l'idée selon laquelle les sols brunifiés de Anikro et de Kahankro sont affectés par la ferrallitisation qui prévaut dans les sols environnants. Cependant, la présence d'illite, 
de chlorite et même de smectites, donne l'idée d'un sol où se déroule toujours un phénomène de bisiallitisation, qui serait soutenu par la richesse de ces sols en minéraux primaires de type feldspath, olivine, calcite auxquels s'ajoute la dolomite et, dont l'altération continue de libérer dans le sol les ions $\mathrm{Ca}^{2+}$ et $\mathrm{Mg}^{2+}$ favorables à ce phénomène. Très souvent, les minéraux primaires et les oxydes néoformés (hématite, boéhmite) observés dans le sol total, sont le fait de ceux contenus dans la fraction argileuse de ces sols. Cette richesse du sol en minéraux primaires ainsi que le caractère argileux de certains oxydes donnent à ces sols l'aspect d'un sol peu évolué, quoique ce caractère minéralogique contribue à enrichir le sol en éléments nutritifs pour les plantes. Ce qui permet de dire que les occurrences minéralogiques des sols brunifiés de Anikro et de Kahankro constituent un atout en termes de fertilité pour la pratique agricole.

\section{REFERENCES}

Aleskeev A, Aleskeeva T, Ostroumov V, Siegert C, Gradusov B. 2003. Mineral transformation in Permafrost- Affected soil, North Kolima Lawland, Russia. Soil Science Socity. American Journal, 67(2): 596-605.

Alvarez-Puebla RA, Aja C, Blasco J, Echeverria JC, Mosquera B, Garrido JJ. 2004. Copper heterogenous nucleation on a palygorskitic clay: an XRD, EXAFS and molecular modeling study. Applied Clay Science, 25(1-2) : 103-110.

Ayari F, Srasra E, Trabelsi-Ayadi M. 2004. Application des modèles de Langmuir et Freundlich aux isothermes d'absorption des métaux lourds par l'argile purifiée. Journal de Physique, 4(122): 229-234.

Ben Hassine H., 2006. Nature minéralogique et rôle nutritionnel des argiles de sols céréaliers en région subhumide à semiaride (Tunisie). Geoscience, 338: 114131.
Black CA, Evans DD, white JL, Ensminger LE, Clark FE. 1965. Methods of Soil Analysis. Amer. Soc. Agron. Inc. Madison: WI. USA.

Brown G. 1961. The X-ray Identification and Crystal Structures of Clay Minerals. The mineralogical Society: London; 17.

Brown DJ, Helmke PA, Clayton MK. 2003. Robust geochimical indice for redox and weathering on a granitic lateritique landscape in Uganda. Geochimica and Cosmochimica Acta, 67(15): 2711-2723.

Campy M, Macaire J. 2003. Erosion, transfert et stockage dans les environnements continentaux. In Géologie de la Surface (2è édn). Dunod : Paris.

Caner L. 2004. Déstabilisation de la gibbsite dans les andosols du massif des Nilgiri en Inde du Sud. Actes des 8è Journées Nationales de l'étude des Sols, 26-28 octobre, Bordeau, 127 p.

Darboux F, Le Bisonnais Y. 2007. Changes in structural stability with soil surface crusting: consequences for erobility estimation. European Journal of Soil Science, 58: 1107-1114.

Doumbia D, Yao KP, Yao NR. 2002. Sciences et Technologies. CM Éditions Éburnie: Abidjan.

Drits VA, Lindgreen H, Sakharov BA, Jacobsen HJ, Zviagina BB. 2004. The detailed structure and origin of clay minerals at the Cretaceous/Tertiary boundary, Stevns Klint (Denmark). Clay Minerals, 39: 367-390.

Drits VA, Lindgreen H, Sakharov BA, Jakobsen HJ, Fallick AE, Salyn AL. 2007. Formation and transformation of mixed-layer minerals by Tertiary intrusives in Cretaceous mudstones, West Greenland. Clays \& Clay Minerals, 55: 260-283.

Duchaufour P. 2001. Introduction à la Science du Sol: Sol, Végétation, Environnement. 
(6 $6^{\mathrm{e}}$ éd) de l'Abrégé de Pédologie. Dunod : Paris.

Feder F. 2001. Dynamique des processus d'oxydo-réduction dans les sols hydromorphes: Monitoring in situ de la solution du sol et des phases solides ferrifères. Thèse de doctorat de l'université de droit, d'économie et des sciences d'Aix-marseille, 83-103.

Grimaldi C. 2004. Hydrochimie de petits bassins versants. Apport à la connaissance du fonctionnement géochimique du sol et de la qualité de l'eau des cours d'eau. Habilitation à diriger les recherches, Université de Renne 1, 79 p.

Holtzapffel T. 1985. Les minéraux argileux : Préparation, analyse diffractométrique et détermination. Société Géologique $d u$ Nord, 12: 95-106.

Horbe AMC, Horbe MA, Suguio K. 2004. Tropical Spodosol in northeastern Amazonas State, Brazil. Geoderma, 119: 55-68.

Hubert F, Caner L, Meunier A, Lanson B. 2009. Advences in characterization of soil clay mineralogy using X-ray diffraction: from decomposition to profile fitting. European Journal of Soil Science, 60: 1093-1105.

Jackson ML. 1969. Soil chemical analysis. Advanced course. Published by Autor, Wisconson, Univ.: Madison, USA.

Kadiebu SK. 2005. Elaboration d'éléments de support dans des dispositifs thermoélectriques multicouches. Thèse de doctorat de l'université de Limoges; 46-47.

Lanson B, Sakharov BA, Claret F, Drits VA. 2009. Diagenetic Smectite-to-illite transition in Clay-rich sediments: a reappraisal of $\mathrm{X}$-ray diffraction results using the Multi-specimen method. American Journal of Science, 309: 476516.
Legros JP. 2007. Enfoncement progressif, autres mécanismes de pédogenèse et organisation spatiale des sols. In Les Grands Sols du Monde. Presse Polytechnique et Universitaire Romande: Lausanne; 95-136.

Mallam IO, Cousin I, Le Bissonnais Y, Quétin P. 2004. Dynamic evolution of the unsaturated hydraulic conductivity of a developing crust. Earth Surface Processes and Landforms, 29: 11311142.

Mc Carty DK, Sakharov BA, Drits VA. 2008. Early clay diagenesis in gulf caost sediments: New insights from XRD profil modeling. Clays and Clay Minerals, 56: 359-379.

Moore DM, Reynolds JRC. 1997. X-ray diffraction and the Identification and Analysis of Clay Minerals. Oxford Univ. Press: New York, Oxford.

Oh NH, Richter DD. 2005. Elemental translocation and loss from three highly weathered soil-bedrock profiles in the southeastern United States. Geoderma, 126(1-2): 5-25.

Pedro G. 2004. Caractéristiques des chapeaux de fer en milieux latéritique cuirassé. Compte Rendu Géosciences, 336: 1473 1480.

Quantin C, Becquer T, Rouiller JH, Berthelin J. 2001. Oxyde weathering and trace metal release by bacterial reduction in a New caledonia Ferralsol. Biogeochemistry, 53: 323-340.

Temgou E, Bitom D, Bilong P, Lucas Y, Pfeifer HR. 2002. Démantèlement de paysages cuirassés anciens en zones forestières tropicales d'Afrique centrale : formation d'accumulations ferrugineues actuelles en bas de pente. Compte Rendu Geosciences, Acad. Des Sciences Paris, 334: 537-543. 
Ulery AL. 2002. Amorphous minerals. Encyclopedia of Soil Science, Marcel Dekkler ; 56-59.

Van Ranst E, De Coninck F. 2002. Evaluation of ferrolysis in soil formation. Eropean Journal of Soil Science, 53(4): 513-519.

Veyret N, Vigneau G. 2002. Géographie physique. Milieu et Environnement dans le Système Terre. A. Colin: Paris.

Viswabaskara V, Gnanan FD, Balasubramanian. 2003. Mullite from clay reactive alumina for insulating substrate application. Applied Clay Science, 25: 29-35.
Yao-Kouamé A. 2008. Etude des sols brunifiés dérivés des matériaux du complexe volcano-sédimentaire de Toumodi en moyenne Côte d'Ivoire. Thèse de Doctorat d'état ès-sciences Naturelles. Université de Cocody, Abidjan/UFR STRM, 219 p.

Yoboué KE, Yao-Kouamé A, Alui KA. 2010. Evolution Pédogéochimique et Minéralogique au Cours de l'altération des Formations du Complexe Volcanosédimentaire d'Anikro et de Kahankro (Toumodi) dans le Centre-Sud de la Côte d'Ivoire. European Journal of Scientific Research, 40(1): 60 -72. 